\title{
A Stability Evaluation of W-band Direct Detection Radiometer
}

\author{
Mikuláš Šostronek, Miroslav Matejček \\ Dept. of Electronics \\ Armed Forces Academy of gen. M. R. Štefánik \\ Liptovský Mikuláš, Slovakia \\ mikulas.sostronek@aos.sk
}

\begin{abstract}
This paper deals with a stability evaluation of a direct detection radiometer based on a model in Matlab Simulink. The model results were compared with a real radiometer output. Based on stability evaluation we try to predict an unstable radiometer output by an easy method. This allows to prolong a time between calibration of the radiometer to reach more precise results during measurement of sensed object.
\end{abstract}

Keywords- Direct Detection Radiometer, system stability, radiometer model.

\section{INTRODUCTION}

At the present time, radiometers are in a large scale used in security applications for a detection of hidden weapons e.g. on airports or in a close checking at the entrance to military bases or nuclear power plants. Up to now, this task has been done by metal detectors; but with the appearance of non metallic weapons as ceramic knives or plastic explosives this method is not very effective.

A radiometer is a very sensitive $\mathrm{RF}$ receiver, which is dedicated for measuring of a noise power that is emitted or reflected from sensed objects. This noise power is an equivalent to noise temperature called a brightness temperature that is measured in Kelvin.

\section{MODEL OF DIRECT DETECTION RADIOMETER}

For an arbitrary system it is necessary to determine its stability. As we published in [1] the model of the direct detection radiometer in Simulink is shown at Figure 1.
This diagram undergo minor updates compared to diagram in [1]. We changed time constants that improves overall precision of simulation that reaches $\pm 0.5 \%$ compared with measured values at the output of the radiometer after its warming-up process (see Figure 2. and 3.).

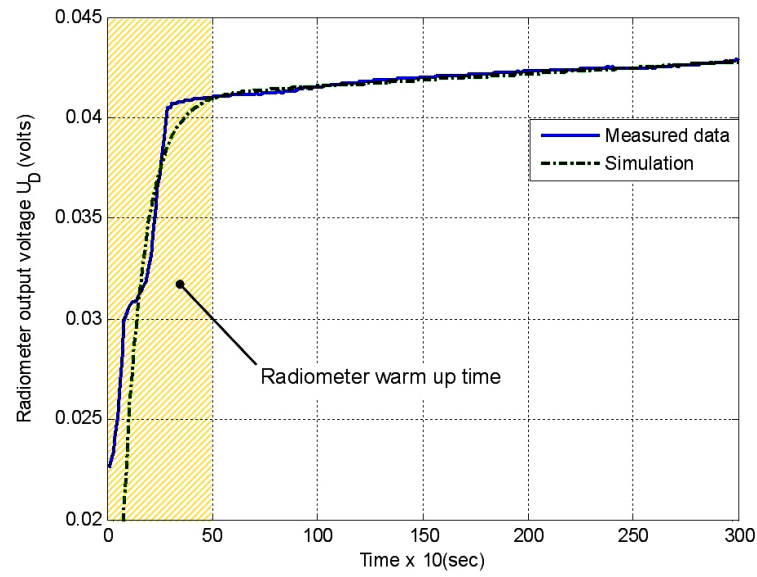

Figure 2. Measured and simulated data from radiometer output.

Moreover, we changed layout of simulation model blocks to be close to its physical realization of real direct detection radiometer.

On the left side of Figure 1 there is realized an input signal emitted by radiating object that is under measurement. This signal is defined by $[3,4,5,6]$

$$
P=k B\left(T_{A}+T_{N}\right) \quad[\mathrm{W}],
$$

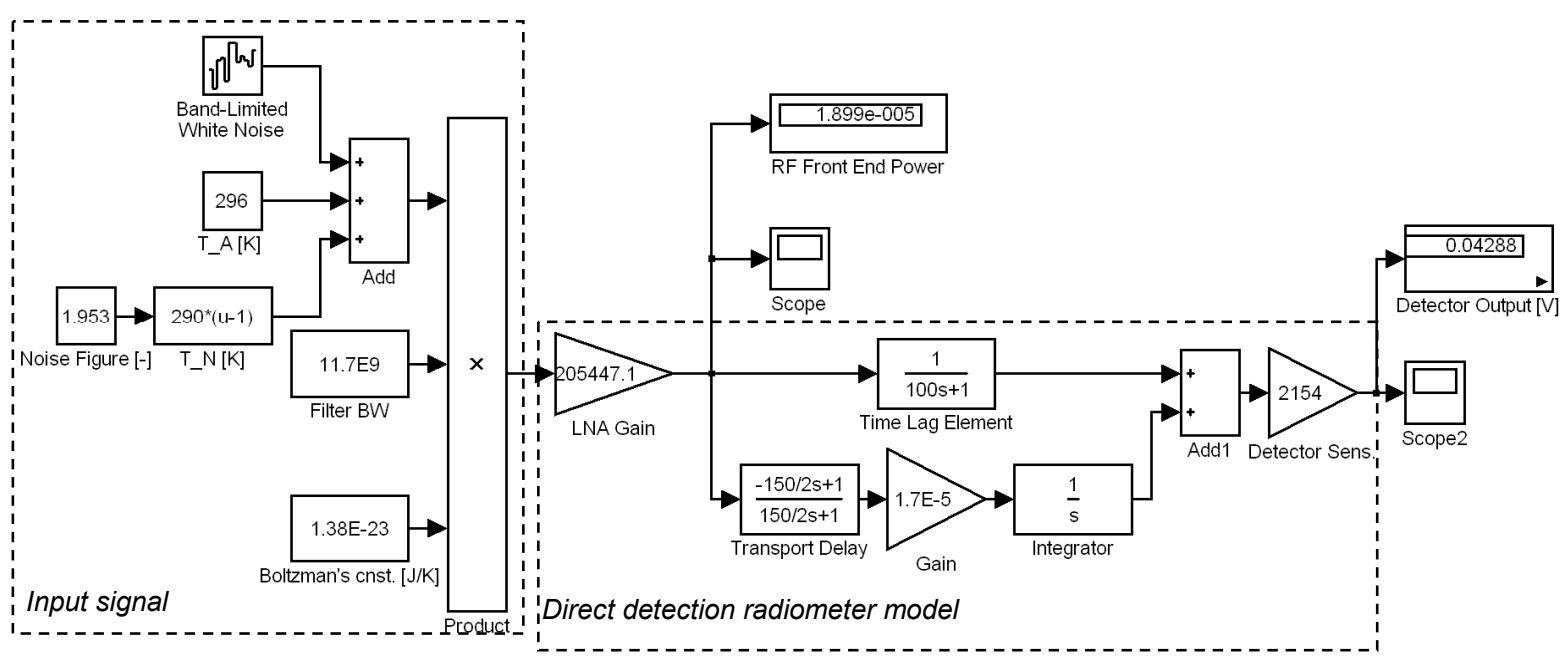

Figure 1. Model of direct detection radiometric module. 
where $k$ is a Boltzmann constant $\left(1.38 \times 10^{-23} \mathrm{~J} / \mathrm{K}\right)$, $B$ is bandwidth of radiometer filter, $T_{A}$ is a noise temperature of antenna and $T_{N}$ is a noise temperature of the radiometer in Kelvins.

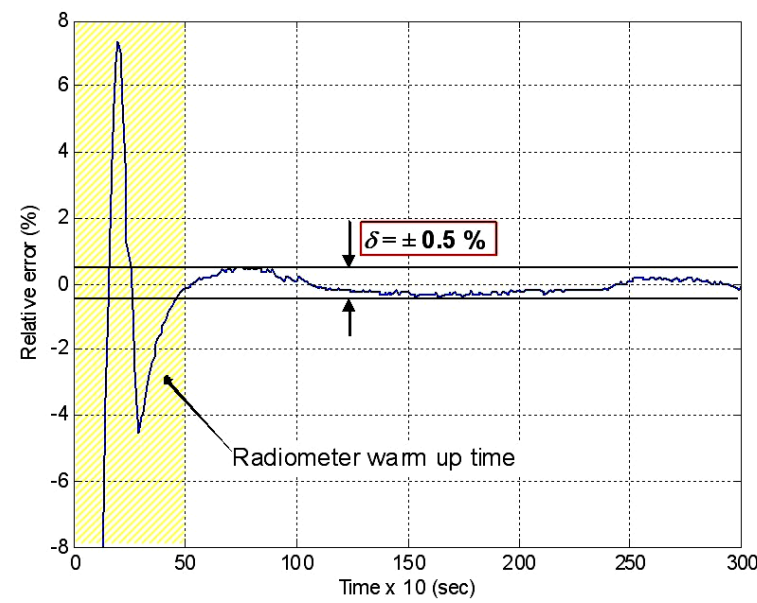

Figure 3. Percentage relative error of simulation.

Right side of diagram represents an RF part of the radiometer. It includes gain $53.127 \mathrm{~dB}$ of two low noise amplifiers (LNA) that is reduced by losses of an $\mathrm{RF}$ filter which is placed between two stage LNAs.

\section{RADIOMETER STABILITY EVALUATION}

In the next text we are going to analyze a stability of the direct detection radiometer. From this reason it is important to determine a transfer function of the radiometer. As we can see, the direct detection radiometer is a single input - single output (SISO) open-loop system without feedback (see Figure 1, blocks in the dashed rectangle on the right side). We reduced block diagram into one transfer function $G(s)$ in a zero-pole-gain form

$$
G(s)=\frac{4513054.249(s+0.01334)\left(s+1.5 \times 10^{-5}\right)}{s(s+0.0133)(s+0.01)} .
$$

As we can see from (2) a characteristic polynomial of transfer function (denominator of transfer function) consist of one integration element $1 / s$ and two phase lag elements $1 /(s+0.0133)$ and $1 /(s+0.01)$, respectively.

To find a stability of system one should compute poles of the transfer function. For SISO transfer functions or zero-pole-gain models the poles are simply denominator roots.

From stability point of view we have three categories in which all linear systems fall:

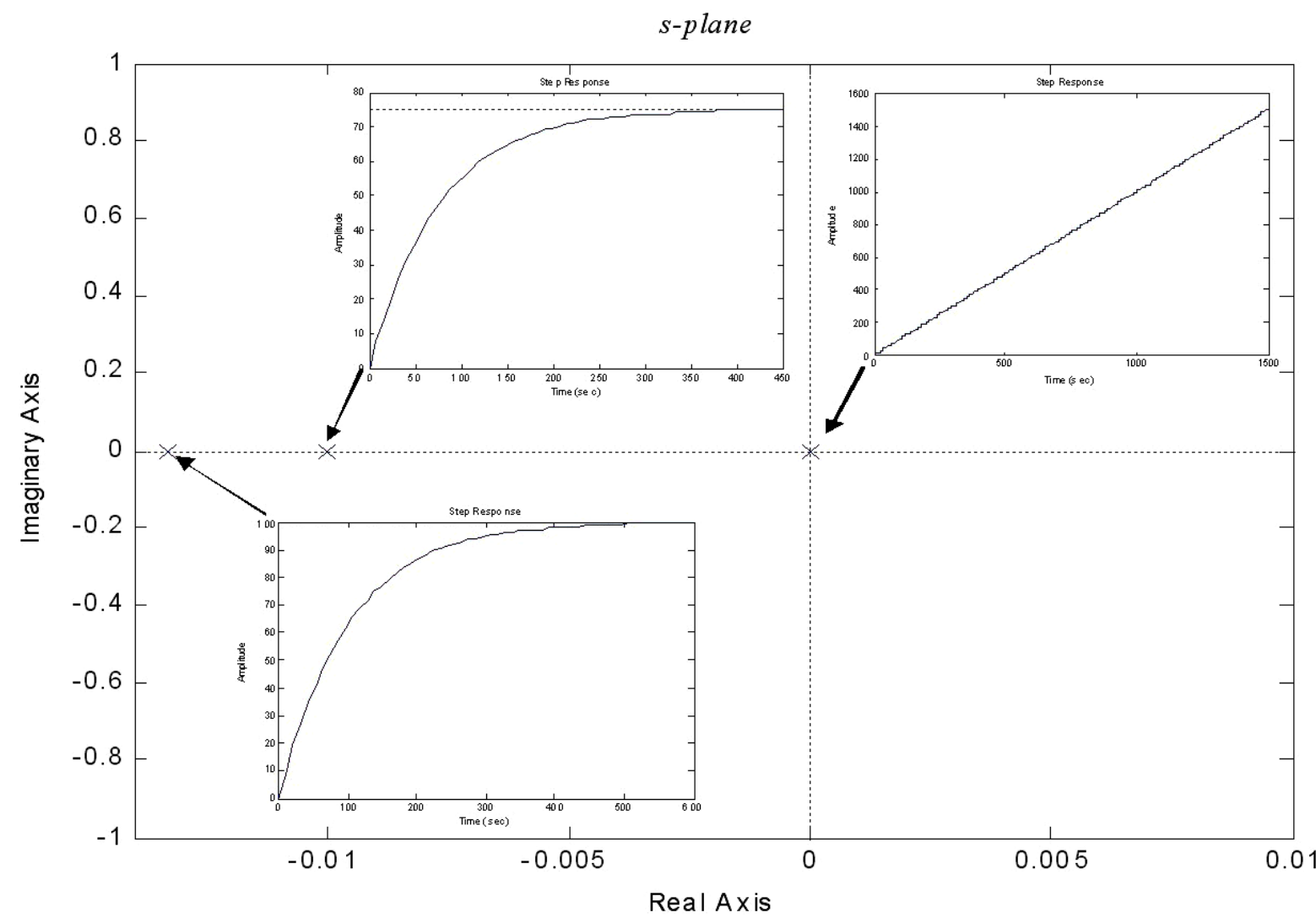

Figure 4. Transfer function poles of the radiometer in $s$-plane.

The next blocks represent a behavior of the radiometer during its warming-up process and the next operation of the radiometer. At the end of a block there is a direct detection diode with a sensitivity of $2154 \mathrm{~V} / \mathrm{W}$ $\approx 2.2 \mu \mathrm{V} / \mathrm{mW}[7]$.
1) If the roots of characteristic polynomial are negative then the initial response to the initial conditions tends to be finite steady value when time $t \rightarrow \infty$. Such systems are called asymptotically stable $[2,9]$.

2) If any root of linear system has a positive real part then its initial response to finite initial conditions 
will be infinite in the magnitude for limit $t \rightarrow \infty$. Such systems are unstable [2, 9].

3) If all roots of a system have a real part less than or equal to zero and all the roots when have zero parts are simple i.e. they are not repeated (multiple) roots. The initial response of the system to finite initial conditions will keep oscillations with a finite amplitude in the limit when time $t \rightarrow \infty$. Such a system is said to by stable but not asymptotically. The output response does not tend to constant steady state magnitude for time $t \rightarrow \infty$. If the poles have a zero real part which are repeated (they are multiple poles with the same imaginary parts that are in real systems conjugate) the initial response of the system to the finite initial conditions leads to output infinity in the limit $t \rightarrow \infty$ and that systems are unstable. Thus only real possibility of two (or more) repeated poles having zero real parts is that all such poles should be at origin (i.e. their imaginary parts should be zero) $[2,9]$.

In our case the poles of transfer function (2) are $p_{1}=0, p_{2}=-0.1333$ and $p_{3}=-0,01$ and they were computed in Matlab. So both poles $p_{2}$ and $p_{3}$ are negative and pole $p_{1}$ is equal to zero. No pole is multiple so according to statement 3) in a text mentioned above the system should be stable but not asymptotically.

As we can see from $s$-plane (Figure 4.) poles $p_{2}$ and $p_{3}$ represent finite step response. However, the pole $p_{1}$ has an infinite step response. This pole involves that a step response of all radiometer is unstable. This is clear from Figure 2 where after the warm-up process of the radiometer its output voltage is almost linearly increased.

The direct detection radiometer is a type of a total power radiometer without down conversion. These radiometers are very sensitive but they are very unstable because of amplifiers gain unstability in the front end of a radiometer $[3,8,10,11]$. From this reason there is a necessity to calibrate this radiometer often to obtain precise results with the measurement of objects brightness temperature. The calibration process is embodied in often comparison brightness temperature between black body temperature (well matched load with known physical temperature) and object sensed by the radiometer antenna. This switching process should be done in the interval of few seconds. In the past, one of the solution was to design this radiometer as a Dicke radiometer. This radiometer has better stability but twice worse sensitivity than TPR radiometers. Nowadays a stability of modern total power radiometers is relatively good thanks to the new low noise amplifiers based on InP HEMT MMIC devices with low noise and a direct detection at the RF front end of the radiometer [12]. With that radiometer its output behavior is quite good predictable that can lead to prolong time that is necessary for the next calibration.

The one possibility to predict output of direct detection radiometer is as it was mentioned above to simulate process of the output of radiometer by simulation in Matlab Simulink.

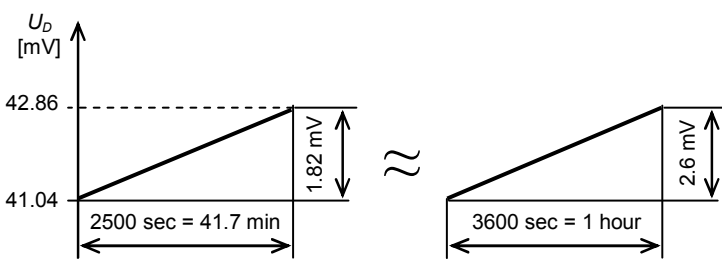

Figure 5. Approximation of radiometer output during measurement and predicted state after 1 hour

However, there is one more easier solution to evaluate radiometer output. As we can see from Figure 2 , the radiometer output after warm-up process is linearly increasing. This can be approximate by a line with the same rate of gradient as the radiometer output

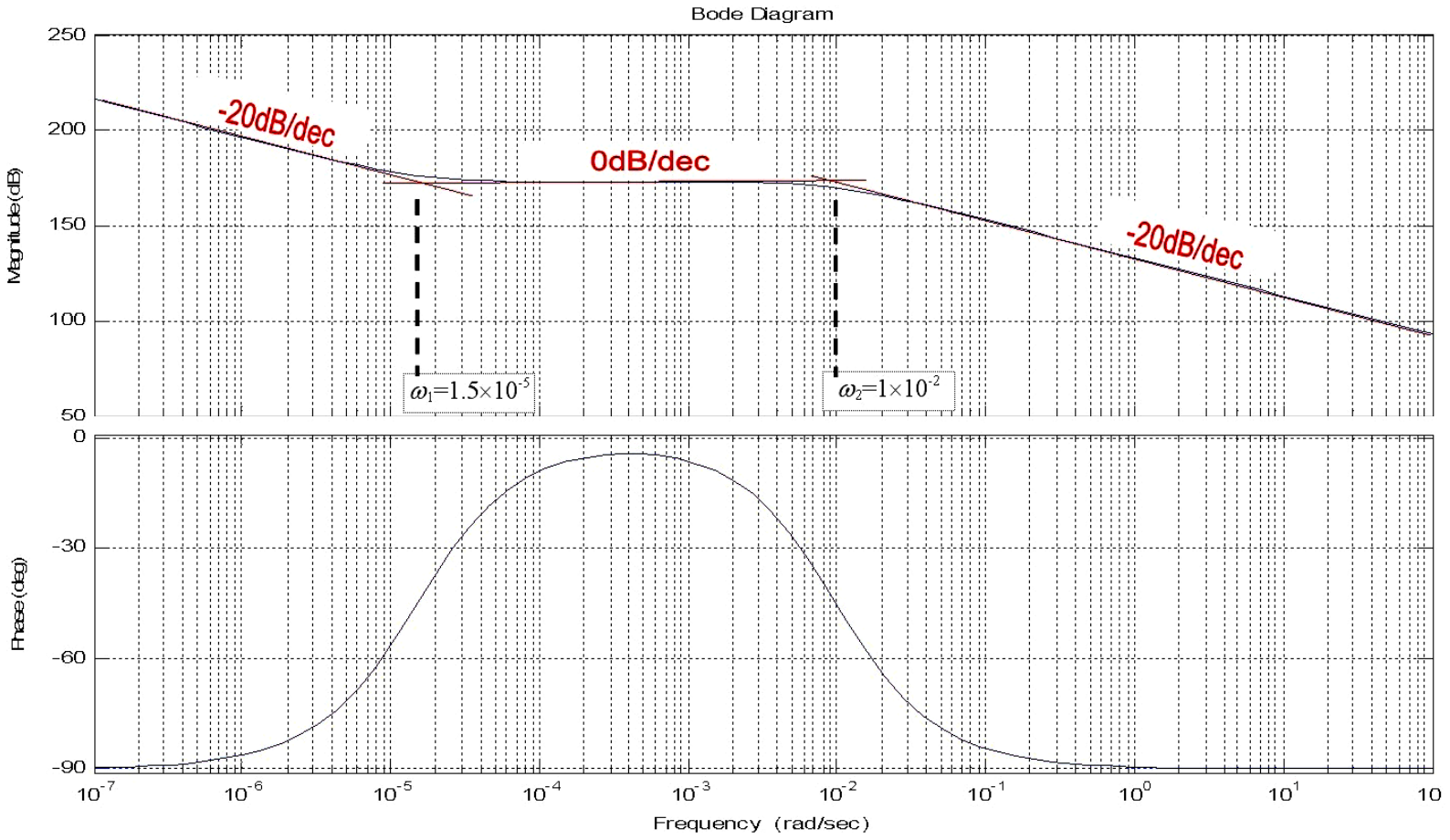

Figure 6. Bode plot of the radiometer model. 
in steady state as it is shown at Figure 5.

As we can see from Figure 5. a gain instability after 1 hour reaches 2,6 mV. From calculated results published in [1] 1 Kelvin resolution of radiometer is equal $0.073 \mathrm{mV}$. So after 1 hour of the radiometer operation the error at its output will be 35.628 Kelvins. After 1 minute of operation the radiometer error will be 0.5947 Kelvins. From this result one can suppose that for assurance of approximately 0.6 Kelvin resolution there is necessity to calibrate in interval less than 1 minute. However, it is possible to increment a measured brightness temperature during the measurement with approximately 0.6 Kelvins per minute and an interval to the next calibration can be extended up to few minutes.

As it twas mentioned above with Matlab simulation in Simulink we can predict the output of the radiometer with less than $0.5 \%$ error during all time of radiometer operation after warm-up process. After re-calculation it means that prediction of the output radiometer is in \pm 1.468 Kelvin range. To compare, very accurate astronomy radiometers has sensitivity of 0.5 up to 1 Kelvin.

\section{FREQUENCY RESPONSE ANALYSIS}

In conclusion we can show a Bode plot at Figure 6 that it was done in Matlab. As we can see in the gain plot (upper in Bode subplot) one can approximate it by asymptotes. The first asymptote inclines with -20 $\mathrm{dB} /$ decade and it is a given by $1 / s$ element (ideal integrator) from transfer function. From the frequency $\omega_{1}=1.5 \times 10^{-5} \mathrm{rad} / \mathrm{sec}$ the asymptote changes to $0 \mathrm{~dB} /$ decade which is caused by influence of $\left(s+1.5 \times 10^{-5}\right)$ element that is phase lead element. This asymptote ends at frequency $\omega_{2}=0.01 \mathrm{rad} / \mathrm{sec}$ where the last phase lag element $(s+0.01)$ causes that asymptote starts to descend with $-20 \mathrm{~dB} / \mathrm{decade}$ inclination. The transport delay element $(s+$ $0.01334) /(s+0.133)$ has no effect on a gain plot and it causes only time shift in step response as it is shown at Figure 2.

The phase plot (lower in Bode subplot) shows behavior of phase when frequency is changed. Phase is -90 degrees during all shown interval (particularly caused by integral and phase lag elements, respectively) except interval between frequencies $\omega_{1}$ and $\omega_{2}$ where phase is 0 degrees. In this interval the radiometer acts as a proportional element with 0 $\mathrm{dB} /$ decade gain and 0 degrees phase shift.

\section{SUMMARY}

As it was mentioned earlier direct detection radiometers are some kind of total power radiometers. They have high sensitivity at the expense of a radiometer stability. In the past, this problem was solved by frequent calibration of the radiometer that leads to prolong time that was necessary to scan object brightness temperature. In the article the stability of the radiometer was evaluated. Based on this analysis a possibility to predict behavior of the direct detection radiometer output by simple method was done. This can allow to prolong time interval between sensing an object under measurement and calibration of radiometer.

\section{REFERENCES}

[1] Šostronek, M. et al., "W-Band Direct Detection Radiometer Model". In: 20th International Conference on Applied Electronics 2015 : Pilsen, 8 - 9 September 2015. - Pilsen : West Bohemian University, 2015. - ISBN 978-80-261-03851. - S. 221-224..

[2] A. Tewari, "Modern Control Design With MATLAB and SIMULINK", John Wiley \& Sons, Ltd. New York, 2002, ISBN 0471496790

[3] N. Skou, D. Le Vine, "Microwave Radiometer Systems. Design and Analysis", Artech House, Norwood, 2006, ISBN: 1-58053-974-2G.

[4] D. Pozar, "Microwave Engineering", 4-th Ed. McGraw- Hill, New York 2011

[5] B. R. Mafhaza, "Introduction to radar analysis", CRC Press, Boca Raton, 2006, ISBN: 0-8493-1879-3.

[6] F. T. Ulaby, R. K. Moore, A. K. Fung, ,Microwave remote sensing: Active and Passive. Volume I, Microwave Remote Sensing, Fundamentals and Radiometry“. Addison-Wesley, Reading, Mass, 1981.

[7] Farran Technology Ltd., "W-band Direct Detection Module With 2 x LNA, 1 x Filter, 1 x Detector", Job No: 1942 Elsinco, datasheet, pp. 1-5, January 2007.

[8] M. Šstronek, Zd. Matoušek, et al., "Experimental verification of radiometer properties", Proceedings on New Trends in Signal Processing 2012, NTSP 2012, Tatranské Zruby, May 2012, pp. 148-153.

[9] R. C. Dorf, R. H. Bishop, "Modern Control Systems" 12-th Edition, Pearson, New Jersey, 2010, ISBN-13: 9780136024583.

[10] T. Jin, Q. Wu, P. Gu, et al. A $150 \mathrm{GHz}$ dual polarization radiometer receiver subsystem. In IEEE International Workshop on Electromagnetics: Applications and Student Innovation Competition (iWEM) 2016 : May 16-18, 2016. New York : IEEE, 2016. ISBN 978-1-4673-8194-9.

[11] Z. Wang, D. Shen, C. Wang et al. Research of $183 \mathrm{GHz}$ fivechannels radiometer receiver subsystem. In IEEE International Workshop on Electromagnetics: Applications and Student Innovation Competition (iWEM) 2016 : May 1618, 2016. New York : IEEE, 2016. ISBN 978-1-4673-8194-9.

[12] Essen, H. et al.: "Concealed Weapon Detection with Active and Passive Millimeterwave Sensors, Two Approaches" [online 02. 06. 2015] http://duepublico.uni-duisburgessen.de/servlets/DerivateServlet/Derivate14694/Final_Papers/GM0004-F.pdf. 